\title{
Effect of interchange instability on magnetic reconnection
}

\author{
W. Lyatsky and M. L. Goldstein \\ NASA Goddard Space Flight Center, 8800 Greenbelt Rd., Greenbelt 20771, MD, USA \\ Correspondence to: W. Lyatsky (wladislaw.lyatsky@nasa.gov) and M. L. Goldstein (melvyn.l.goldstein@ nasa.gov)
}

Received: 31 August 2011 - Revised: 3 November 2012 - Accepted: 30 November 2012 - Published: 13 June 2013

\begin{abstract}
We present here the results of a study of interacting magnetic fields that involves a force normal to the reconnection layer. In the presence of such force, the reconnection layer becomes unstable to interchange disturbances. The interchange instability results in formation of tongues of heated plasma that leaves the reconnection layer through its wide surface rather than through its narrow ends, as is the case in traditional magnetic reconnection models. This plasma flow out of the reconnection layer facilitates the removal of plasma from the layer and leads to fast reconnection. The proposed mechanism provides fast reconnection of interacting magnetic fields and does not depend on the thickness of the reconnection layer. This instability explains the strong turbulence and bidirectional streaming of plasma that is directed toward and away from the reconnection layer that is observed frequently above reconnection layers. The force normal to the reconnection layer also accelerates the removal of plasma islands appearing in the reconnection layer during turbulent reconnection. In the presence of this force normal to the reconnection layer, these islands are removed from the reconnection layer by the "buoyancy force", as happens in the case of interchange instability that arises due to the polarization electric field generated at the boundaries of the islands.
\end{abstract}

\section{Introduction}

The importance of magnetic reconnection in astrophysics and heliospheric physics is widely recognized. However, despite numerous studies and a remarkable progress, there is yet insufficient understanding of the real cause for fast reconnection rate that is inferred from experimental data.

The first solution to magnetic reconnection was proposed by Sweet (1958) and Parker (1957), who considered the reconnection of two oppositely directed magnetic fields as a two-dimensional incompressible magnetohydrodynamic MHD problem and estimated the reconnection rate. The traditional Sweet-Parker model is based on the assumption that reconnection occurs in a thin layer with the length $2 L$ and thickness $2 \delta$ (see Fig. 1). The plasma is assumed to be incompressible. From mass conservation, they obtained $\rho V_{z} L=\rho V_{x} \delta$ where $\rho$ is the plasma density, and $V_{z}$ and $V_{x}$ are the plasma velocities across and along the reconnection layer, respectively. The velocity $V_{x}$ along the x-axis may be estimated from energy conservation that gives $(1 / 2) \rho V_{x}^{2}=$ $B^{2} / 8 \pi$, which results in $V_{x} \approx B /(4 \pi \rho)^{1 / 2}=V_{\mathrm{A}}$, where $V_{\mathrm{A}}$ is the Alfvén velocity in ambient plasma. Then the relation $\rho V_{z} L=\rho V_{x} \delta$ may be rewritten as

$V_{z}=V_{\mathrm{A}} \delta / L$.

This relation plays a very important role in the classical theory of reconnection. It shows that the reconnection rate $V_{z}$ is restricted by the value of the ratio of the thickness of the reconnection layer to its length. Due to large sizes of interacting magnetic fields, this ratio in space physics is usually very small, which strongly reduces the reconnection rate. However, that contradicts the experimental results which show that, in fact, the reconnection rate is often much higher.

To resolve this inconsistency, Petschek (1964) suggested that the reconnection can occur not along the entire layer but on its shorter length, while the tension of reconnected magnetic fields at the edges of the reconnection layer will accelerate and expel plasma along the layer; this leads to faster reconnection. The Petschek model including the X-line was widely accepted as a reasonable solution until numerical simulations by Biskamp (1986), Uzdensky and Kulsrud (2000), Dorelli and Birn (2003), and others showed that this model is unstable (at least, for uniform resistivity of plasma) and quickly converts to the slow but stable Sweet-Parker model. This result inspired researchers to examine other reconnection mechanisms involving anomalous resistivity of 


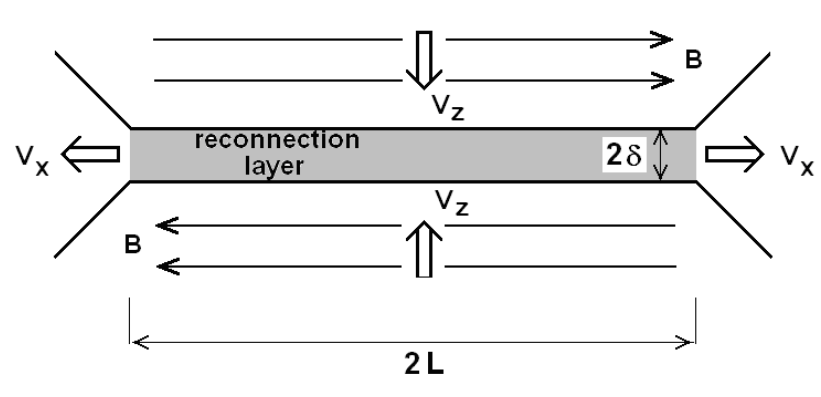

Fig. 1. The Sweet-Parker model. The magnetic fields, $\boldsymbol{B}$, have opposite directions above and below a thin reconnection layer. Plasma is frozen into the magnetic fields and enters the reconnection layer at the velocity $V_{z}$ and moves out of it at the velocity $V_{x}$.

plasma that might stabilize the Petschek mechanism (Kulsrud, 2001), turbulent reconnection (e.g., Matthaeus et al., 1984; Lazarian and Vishniac, 1999), Hall reconnection (e.g., Birn et al., 2001; Shay et al., 2001), and others.

Turbulent reconnection, investigated by Matthaeus et al. (1984), Matthaeus and Lamkin (1986), Lazarian and Vishniac (1999), and others, produces multiple X-lines, the separation of the reconnection layer into shorter segments, and the formation of plasma islands along the current sheet. Since separation of the reconnection layer into shorter parcels reduces the effective length of this layer, it was expected that this process can increase the reconnection rate. However, piling-up plasma islands in the reconnection region can prevent fresh plasma and magnetic field from entering the reconnection layer, which reduces the reconnection rate. And indeed, while some researchers (e.g., Loureiro et al., 2007) found that turbulence leads to increasing the reconnection rate, others (e.g., Shepherd and Cassak, 2010) found that turbulent reconnection leads to an insignificant increase in the reconnection rate only.

Another approach, the Hall reconnection, has been investigated numerically by using two-fluid simulations (e.g., Birn et al., 2001; Shay et al., 2001). The two-fluid approach leads to different thicknesses of the ion and electron layers. On the length scales shorter than the ion inertial length $d_{\mathrm{i}}=c / \omega_{\mathrm{pi}}$, where $c$ is the light velocity and $\omega_{\mathrm{pi}}$ is the ion plasma frequency, ions become demagnetized and decouple from electrons (e.g., Birn et al., 2001; Dorelli and Birn, 2003; Yamada et al., 2010; Huang et al., 2011). In this case, the Hall term in the Ohm's law becomes important, which results in a significant increase of reconnection rate. The simulations by Shay et al. (2004) and Cassak and Shay (2008) showed, for instance, that the reconnection rate in this case can increase up to about $0.1 V_{\mathrm{A}}$, where $V_{\mathrm{A}}$ is the Alfvén velocity. The cause for fast reconnection rate in the Hall approximation, when the thickness of the layer between two oppositely directed magnetic fields becomes less than the ion inertial length $d_{\mathrm{i}}$, is suggested to be the spontaneous transition from slow SweetParker reconnection to fast Petschek-like reconnection (e.g.,
Dorelli and Birn, 2003; Cassak et al., 2005; Huang et al., 2011).

Although the results, obtained from two-fluid collisionless MHD models of the Hall reconnection, are impressive, the recent studies showed that the standard model of Hall reconnection encounters some problems. Daughton et al. (2006) showed, for example, that there is a "significant inconsistence" between the results obtained from kinetic simulations and MHD simulations of the Hall reconnection. Particularly, they showed that the formation of a "bottleneck" for outflow electrons in the electron diffusion region can substantially reduce the reconnection rate. Another problem is the effect of both embedded turbulence (as mentioned above) and internal plasmoid instability (e.g., Loureiro et al., 2007; Cassak and Shay, 2008; Daughton et al., 2009) within the reconnection layer, which leads to the formation of magnetic/ plasma islands (plasmoids). Recently, Huang et al. (2011) also showed that the transition to Hall reconnection may result in the formation of not a single $\mathrm{X}$-point but several $\mathrm{X}$-points in the reconnection layer that also lead to the formation of plasmoids, which link the Hall reconnection and turbulent reconnection. The effect of plasmoids on reconnection rate will be discussed later.

Another way of solving the problem was proposed by Lyatsky and Goldstein (2010), who showed that reconnection rate can significantly increase in the presence of a force normal to the reconnection layer (it may be a gravitational or some other force). In this case, the reconnection layer becomes unstable against Rayleigh-Taylor (interchange) instability that results in formation of Bénard convection over the reconnection layer, which can provide for fast removal of plasma piling up in this layer, not along the reconnection layer but normal to it. Such a scenario results in fast reconnection that does not depend on the length of reconnection layer.

The mechanism of fast reconnection in this case is shown schematically in Fig. 2. During magnetic reconnection, plasma with frozen-in magnetic field enters into the reconnection layer. In the process of reconnection, the magnetic field annihilates in this layer, and energy of the magnetic field goes into heating the plasma. The heated plasma (especially ions) piles up in a diffusion region surrounding this layer (as in the case of the Hall reconnection). For simplicity, we will consider this region as a part of the reconnection layer, though the difference between these two regions sometimes may be important.

In the presence of a force normal to the reconnection layer, this situation is known to be instable to interchange perturbations, which leads to a wave-like structure at one or both boundaries of the reconnection layer. The growth of these disturbances may result in the formation of the Bénard convection cells with the tongues of heated plasma moving out from the reconnection layer. This process supports fast reconnection, and it does not depend on the length of reconnection region. 


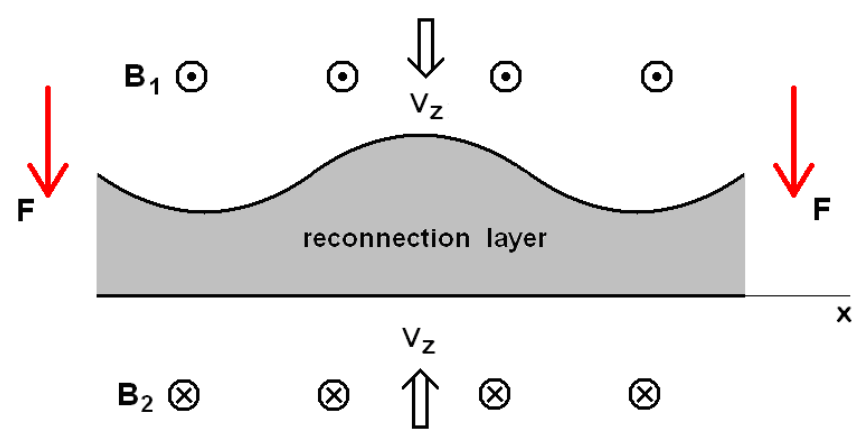

Fig. 2. Generation of interchange instability on upper boundary of the diffusion region surrounding the reconnection layer in the presence of a force $\boldsymbol{F}$, normal to the layer. $\boldsymbol{B}_{1}$ and $\boldsymbol{B}_{2}$ are the magnetic fields above and below the reconnection layer. $V_{z}$ is the initial velocity of plasma with a frozen-in magnetic field. The violated upper boundary shows an interchange disturbance.

The instability in the gravitational field can develop when plasma in the reconnection layer and diffusion region becomes either lighter or heavier than ambient plasma. If plasma in this layer becomes lighter, the buoyancy force is directed upward and the instability develops at upper boundary of the layer, but if plasma becomes heavier, the buoyancy force is directed downward and the instability develops at its lower boundary. So, the instability can develop in both cases if reconnection violates the balance of forces acting on plasma.

In this study, we consider both the real and effective gravitational forces acting on heated plasma in the magnetic field. The effective gravitational force is associated with the diamagnetic force, $-n \mu \nabla B$, where $n$ is plasma density, $B$ is the magnetic field, and $\mu$ is the magnetic moment of particles $\left(\mu=w_{\perp} / B\right.$ where is $w_{\perp}$ is the particle energy across the magnetic field $B$ ). This force is directed against the $\nabla B$. Motion of plasma in this case can also be described as the $\boldsymbol{E} \times \boldsymbol{B}$ plasma drift in the electric and magnetic fields. If heated plasma in the diffusion region surrounding the reconnection layer moves upward (against the gradient of the outer magnetic field), the interchange instability develops on the upper boundary of this region (see, e.g., Sonnerup and Laird, 1963; Lyatsky and Sibeck, 1997).

The purpose of this paper is to present the results of our studies of the effect of interchange instability on the reconnection rate. We will show that the existence of a force normal to the reconnection layer significantly facilitates the removal of plasma from the reconnection layer, which opens the way to fast reconnection.

\section{Rayleigh-Taylor (interchange) instability}

Before considering the role of the Rayleigh-Taylor (R$\mathrm{T}$ ) instability in magnetic reconnection, we consider some

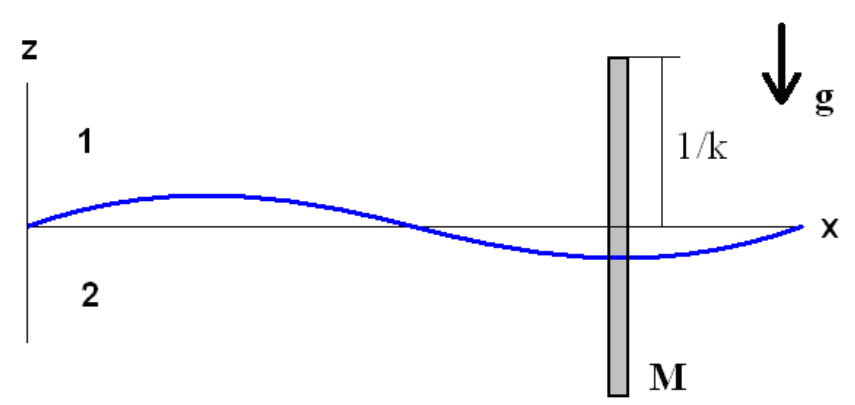

Fig. 3. A schematic for finding the growth rate of R-T instability. Region 1 is related to a heavier fluid, region 2 to a lighter fluid. The undisturbed boundary between two fluids is located in the $x-y$ plane (normal to the figure plane). The gravitational acceleration $\boldsymbol{g}$ is downward. $M$ is the mass of a fluid element having the height equal to $1 / k$ where $k$ is the wave number.

important features of this instability. The R-T instability is based on a well-known phenomenon that when a heavy fluid is placed above a lighter fluid in a gravitational field, the situation is unstable. The important condition for initiating the R-T instability is, as mentioned above, the existence of a force normal to the interface between the two fluids. It may be the gravitational force, the inertial force resulting from deceleration of plasma moving toward or out from the reconnection layer, the force due to the curvature of magnetic field lines, and others (see also Winske, 1996). The R-T instability may develop both at the magnetopause, following compression by the solar wind dynamic pressure (Gratton et al., 1996), and in the magnetotail (Pritchett and Coroniti, 2010; Guzdar et al., 2010; Lapenta and Bettarini, 2011). Thus, there are a large variety of situations when the R-T instability can occur. By considering the instability of a system with a force normal to the interface, an effective gravitational acceleration is often introduced that allows for describing the different instabilities using the same or similar equations. We also will use this simplifying approach.

To better understand the features of the R-T instability, first we will derive the growth rate of this instability from simple physical considerations using a fluid-element approach (e.g., Frank-Kamenetsky, 1964; Piriz et al., 2006; Bret, 2011). First, we consider the R-T instability on the boundary of two fluids with no magnetic field.

Let us consider two incompressible fluids in a gravitational field with the gravitational acceleration $\mathbf{g}$ (Fig. 3). The fluids are separated by a thin interface in the $x-y$ plane. The plasma density above the interface (region 1 ) is $\rho_{1}$ and below the interface (region 2) is $\rho_{2}$, where $\rho_{1}>\rho_{2}$.

To solve the problem, we use the equation of motion. In equilibrium, the pressures below and above the interface are equal. Disturbances of the interface can develop along the $\mathrm{z}$-axis. The instability violates pressure balance on the interface. The force acting at the interface is $\Delta p=p_{1}-p_{2}$, where 
$p_{1}$ and $p_{2}$ are the pressures above and below the interface, respectively. Then the motion equation for a fluid element, shown in Fig. 3 by the vertical strip, may be written as

$M \frac{\partial^{2} z^{\prime}}{\partial t^{2}}=\left(\rho_{1}-\rho_{2}\right) g z^{\prime}$,

where $M$ is the mass of the fluid element shown in Fig. 3 by the vertical strip, which is accelerated by the difference of pressures $\Delta p=p_{1}-p_{2}$, and $z^{\prime}$ is the disturbance of the interface position. For incompressible fluid, div $V=0$ (where $V$ is the velocity of the fluid) and the flows above and below the interface are potential. Then we suppose that the perturbations have the form of $\exp (-i \omega t+i k x \mp k z)$, where $\omega$ is the complex frequency, $k$ is is the wave number, and the minus/plus signs ahead of $k z$ are related to the upper and lower fluids, respectively. This expression describes waves propagating along the $\mathrm{x}$-axis and decaying exponentially along the z-axis.

In Fig. 3, the characteristic half-height of the fluid element is $1 / k$ and the mass of this element (equal to the sum of masses above and below the interface) is $M=\left(\rho_{1}+\rho_{2}\right) / k$ (see also Piriz et al., 2006). Then from Eq. (2) we obtain

$-\omega^{2}=\frac{\rho_{1}-\rho_{2}}{\rho_{1}+\rho_{2}} k g>0$.

From Eq. (3) we obtain the well-known expression (e.g., Chandrasekhar, 1961) for the growth rate of the R-T instability

$\gamma=\operatorname{Im} \omega=\left(\frac{\rho_{1}-\rho_{2}}{\rho_{1}+\rho_{2}} k g\right)^{1 / 2}$.

\subsection{Account for the magnetic field}

Let us consider the R-T instability (in this case, it is usually called the interchange instability) for two magnetized plasmas with different densities and different magnetic fields, immersed into a gravitational field with the gravitational acceleration $\boldsymbol{g}$. The same as earlier, we suppose the fluids to be incompressible and separated with a thin interface in the plane x-y. The "lighter" fluid 2 is located below (Fig. 4); the terms "lighter" and "heavier" fluids correspond to the effective buoyancy force acting on heated plasma in the magnetic field.

The presence of the magnetic fields parallel to the interface can lead to appearance of the magnetic tension force, $(1 / 4 \pi)(\boldsymbol{B} \cdot \nabla) \boldsymbol{B}$. Adding this force to the right side of Eq. (2) in the case when the magnetic fields $\boldsymbol{B}_{1}$ and $\boldsymbol{B}_{2}$ have the same or opposite directions yields the following expression for the growth rate of the instability:

$\gamma^{2}=\frac{1}{\rho_{1}+\rho_{2}}\left(k g\left(\rho_{1}-\rho_{2}\right)-k^{2} \frac{B_{1}^{2}+B_{2}^{2}}{2 \pi} \cos ^{2} \vartheta\right)$,

where $B_{1}$ and $B_{2}$ are magnetic fields in the upper and lower regions, respectively, and $\vartheta$ is the angle between the

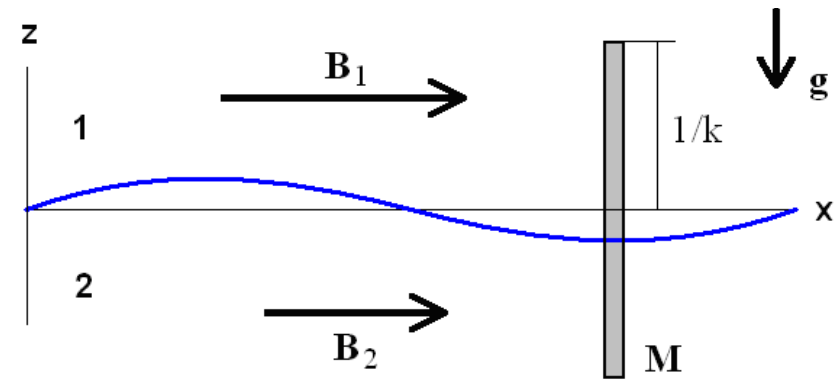

Fig. 4. A schematic to explanation of the interchange instability in magnetized plasma. The numbers 1 and 2 are related to the heavier and lighter fluids, respectively. The undisturbed boundary between two fluids is in the $\mathrm{x}-\mathrm{y}$ plane; $\boldsymbol{g}$ is the effective gravitational acceleration, $M$ is the mass of a fluid element with the half-height $1 / k$ where $k$ is the wave number. $\boldsymbol{B}_{1}$ and $\boldsymbol{B}_{2}$ are the magnetic fields in two media. The instability develops better when the $\boldsymbol{k}$-vector is directed across the magnetic field as in Fig. 2.

magnetic field and the wave vector, $\boldsymbol{k}$. If $j \boldsymbol{B}_{1} j=j \boldsymbol{B}_{2} j$, this expression is consistent with that obtained by Chandrasekhar (1961, p. 466). It may also be rewritten in the following form

$\gamma^{2}=\frac{\rho_{1}-\rho_{2}}{\rho_{1}+\rho_{2}} k g-k^{2} V_{\mathrm{Am}}^{2} \cos ^{2} \theta$,

where $V_{\mathrm{Am}}=B /\left(2 \pi\left(\rho_{1}+\rho_{2}\right)\right)^{1 / 2}$ is the average Alfvén velocity for two media.

Equation (6) shows that the instability may be suppressed by magnetic tension (the last term on the right side of this equation) when the wave vector is close enough to the direction of the magnetic field. However, the magnetic field does not reduce the instability growth rate when the $\boldsymbol{k}$-vector is across the magnetic field. As seen from Eqs. (5)-(6), for small $k$ the growth rate of the instability $\left(\gamma^{2}\right)$ increases linearly with the wave number $k$, achieves its maximum, and decreases to zero at $k=k_{\mathrm{c}}$, where $k_{\mathrm{c}}$ is a critical value of $k$ when the growth rate, $\gamma$, is zero:

$k_{\mathrm{c}}=\frac{2 \pi g\left(\rho_{1}-\rho_{2}\right)}{B^{2} \cos ^{2} \vartheta}$.

The critical wave number, $k_{\mathrm{c}}$, shows the interval of angles, $\vartheta$, in which the instability develops; as seen from Eq. (7), $k_{\mathrm{c}}$ increases with increasing the angle between the $\boldsymbol{k}$-vector and $\boldsymbol{B}$. The growth rate also increases and reaches its maximum for $\vartheta= \pm \pi / 2$ when the $\boldsymbol{k}$-vector is orthogonal to the magnetic field $\boldsymbol{B}$ (see also Jun et al., 1995).

Among other effects which can influence the interchange instability, we mention plasma compressibility and the finite Larmor radius. Above, we considered the growth rate of interchange instability in incompressible fluids. In the case of plasma, this approach is not quite correct. However, both numerical and analytical studies of the effect of plasma compressibility on interchange instability (e.g., Jun et al., 1995; 
Livescu, 2005) showed that the compressibility insignificantly affects its growth rate; moreover, Liberatore and Bouquet (2008) found that compressibility has rather a destabilizing effect on instability development. The finite Larmor radius (FLR) effect on interchange instability was investigated first by Roberts and Taylor (1962), who demonstrated that this effect can stabilize the instability in a short-wavelength limit $k L_{n} \gg 1$, where $k$ is the wave number and $L_{n}$ is the density gradient scale length. Huba (1996) and Winske (1996) confirmed the stabilization effect. Huba (1996) also suggested that, since the FLR leads to the drift velocity of developing perturbations proportional to the diamagnetic drift velocity, this may lead to phase mixing the unstable perturbations within a growth period, which may stabilize the instability. This effect may be significant on short waves.

\section{Reconnection and Rayleigh-Taylor (interchange) instability: geometry and solving the problem}

In this section, we show that magnetic reconnection in the presence of a force normal to the reconnection layer leads to developing R-T (interchange) instability of the heated reconnection layer. In the late stage, the instability results in the formation of tongues of heated plasma, extending normally to the reconnection layer, so that the heated plasma leaves this layer not through its narrow ends but through its wide surface. This facilitates fast removal of plasma from this layer, which leads to fast reconnection. In contrast to the classical theory of reconnection, this mechanism provides for fast reconnection of interacting magnetic fields independent of the sizes of the reconnection region.

This problem may be considered in planar geometry with a vertically directed gravitational acceleration $\boldsymbol{g}$ acting on plasma (Fig. 5). When the distance between the oppositely directed magnetic fields becomes small enough, the magnetic fields "reconnect". This leads to formation of the reconnection layer (region 2) filled with hot plasma.

The reconnection layer in Fig. 5 consists of hot plasma and is located below the ambient colder plasma, located in the gravitational field. This is the classical case of plasma distribution, unstable against interchange perturbations (see Sect. 2)

To estimate the growth rate of interchange instability, we use conservation of mass and energy. Assuming for simplicity that the widths of downward and upward plasma flows are equal yields

$\rho_{1} V_{1}=\rho_{2} V_{2}$,

where $\rho_{1}$ and $\rho_{2}$ are the plasma densities in the two regions, and $V_{1}$ and $V_{2}$ are the vertical velocities of plasmas entering and leaving the reconnection layer, respectively. The indices 1 and 2 denote the regions 1 (surrounding plasma) and 2 (reconnection layer), respectively. For simplicity, the electron mass is neglected.

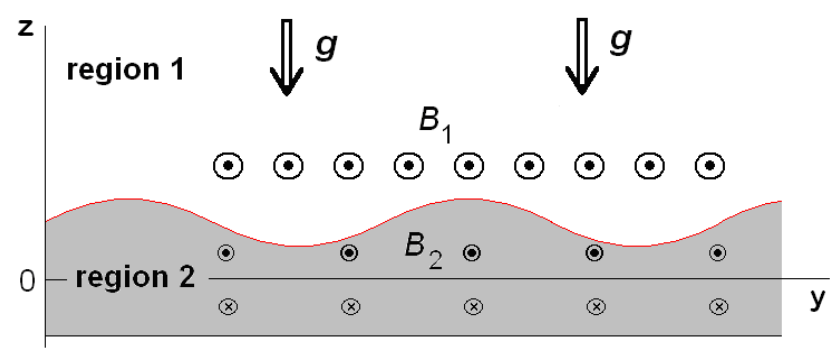

$\otimes \otimes \otimes \otimes \otimes \otimes \otimes \otimes$

Fig. 5. The planar geometry used for solving the problem. Regions 1 and 2 are the surrounding plasma and reconnection layer, respectively. Magnetic fields $\boldsymbol{B}_{1}$ and $\boldsymbol{B}_{2}$ are perpendicular to the plane of the figure, and related to the ambient plasma and the reconnection layer, respectively; $\boldsymbol{g}$ is the effective gravitational acceleration. The boundary between regions 1 and 2 shows an interchange disturbance on the reconnection layer upper boundary. Only the upper part of the disturbed reconnection region is shown.

The energy flux along a streamline for incompressible magnetized plasma may be written in the following form (e.g., Somov, 2006; Schnack, 2009):

$$
\begin{aligned}
\boldsymbol{\Phi} & =\left(\rho \frac{V^{2}}{2}+p\right) \boldsymbol{V}+\frac{c}{4 \pi}(\boldsymbol{E} \times \boldsymbol{B}) \\
& =\left(\rho \frac{V^{2}}{2}+p\right) \boldsymbol{V}+\frac{1}{4 \pi} B^{2} \boldsymbol{V},
\end{aligned}
$$

where the first two terms on the right side show fluxes of kinetic energy of plasma, $(1 / 2) \rho V^{2}$, and internal energy (or plasma pressure, $p$ ), respectively, while the last term shows the flux of electromagnetic energy, described by the Poynting vector $(c / 4 \pi) \boldsymbol{E} \times \boldsymbol{B}$, where $\boldsymbol{E}$ and $\boldsymbol{B}$ are the vectors of the electric and magnetic fields. We accounted here that the velocity $\boldsymbol{V}=c(\boldsymbol{E} \times \boldsymbol{B}) / B^{2}$, so that in our case $(\boldsymbol{V} \perp \boldsymbol{B})$ the Poynting vector is equal to $(1 / 4 \pi) B^{2} V$. Then, the Eq. (9) energy conservation in combination with Eq. (8) may be written in the form

$$
\frac{p_{1}+2 p_{\mathrm{m} 1}}{\rho_{1}}+\frac{V_{1}^{2}}{2}=\frac{p_{2}+2 p_{\mathrm{m} 2}}{\rho_{2}}+\frac{V_{2}^{2}}{2},
$$

where $p_{1}$ and $p_{2}$ are plasma pressures, and $p_{\mathrm{m} 1}=B_{1}^{2} / 8 \pi$ and $p_{\mathrm{m} 2}=B_{2}^{2} / 8 \pi$ are magnetic field pressures. Note that the gravity term does not enter Eq. (10) as both sides of this equation are related to the same altitude, where particles have the same gravitation potential.

Assuming also that in region 1 the magnetic pressure $p_{\mathrm{m} 1}=B_{1}^{2} / \pi \gg p_{1}$ while in region 2 the plasma pressure $p_{2} \gg p_{\mathrm{m} 2}$ yields

$\frac{2 p_{\mathrm{m} 1}}{\rho_{1}}+\frac{V_{1}^{2}}{2} \approx \frac{p_{2}}{\rho_{2}}+\frac{V_{2}^{2}}{2}$.

This equation shows that the energy flux, entering the reconnection layer, is spent on the increase in pressure $p_{2}$ of 
plasma, leaving the reconnection layer, and its acceleration to the velocity $V_{2}$. Taking into account $2 p_{\mathrm{m} 1} / \rho_{1}=B_{1}^{2} / 4 \pi \rho_{1}=$ $V_{\mathrm{A} 1}^{2}$, where $V_{\mathrm{A} 1}$ is the Alfvén velocity in region 1 , and neglecting (like in the Sweet-Parker model) the term $p_{2} / \rho_{2}$ (see above), we obtain the rough estimate for outflow velocity $V_{2} \approx 1.4 V_{\mathrm{A} 1}$. Then accounting for Eq. (8) and neglecting the factor of 1.4 yields the following simple estimate for the reconnection rate:

$V_{1} \approx\left(\rho_{2} / \rho_{1}\right) V_{\mathrm{A} 1}$.

In the case $\rho_{1}=\rho_{2}$ as in the Sweet-Parker (S-P) model, we obtain $V_{1} \approx V_{\mathrm{A} 1}$, which in contrast to S-P model does not contain the small scaling parameter $\delta / L$ and, therefore, provides for fast reconnection. We remind that by obtaining Eq. (12), we neglected the plasma outflow along the x-axis, responsible for the Sweet-Parker mechanism, since it provides only a small increase in the reconnection rate given by Eq. (12). Therefore in limit $g \rightarrow 0$, Eq. (12) does not lead to the interchange instability and reconnection.

Note that Eq. (12) is obtained from the same equations as the S-P solution $V_{z}=V_{\mathrm{A}} \delta / L$. However, whereas the reconnection rate in the S-P mechanism depends on the small factor $\delta / L$, in our mechanism it depends on the factor $\rho_{2} / \rho_{1}$, which is insignificantly different from 1 , providing a much faster reconnection rate in our model. Note also that although the value $g$ indeed does not enter directly Eq. (12), it enters Eq. (6) that derives the growth rate of interchange instability, which in turn affects the reconnection rate. For more details, see also Sect. 5.

Thus, the reconnection layer of heated plasma, located under the surrounding colder plasma in the field of a force normal to the reconnection layer, may be unstable against interchange instability. In steady-state conditions, interchange instability results in the formation of Rayleigh-Bénard convection, which is shown schematically in Fig. 6. The convection includes downward and upward plasma flows. Surrounding plasma and the magnetic field enters the reconnection layer, where a significant portion of inflow magnetic field dissipates. This leads to heating of plasma in the reconnection layer and its outflow back (upward).

Above we considered interchange perturbations, oriented along the magnetic field, when the angle $\vartheta$ between the $\boldsymbol{k}$ vector and the magnetic field $\boldsymbol{B}$ is close to $\pm \pi / 2$. This case is related to the highest rate of instability and the highest reconnection rate. Therefore, the perturbations, oriented along the magnetic field, are especially important for study.

Thus, the difficulty in explanation of fast reconnection in the standard Sweet-Parker model is related to the necessity of the removal of plasma from the reconnection layer through its narrow ends, which strongly reduces the reconnection rate for large sizes of interacting magnetic fields. Here we considered another approach, which facilitates the removal of plasma from the reconnection layer that provides for fast reconnection. The main results of this approach may be described as follows:

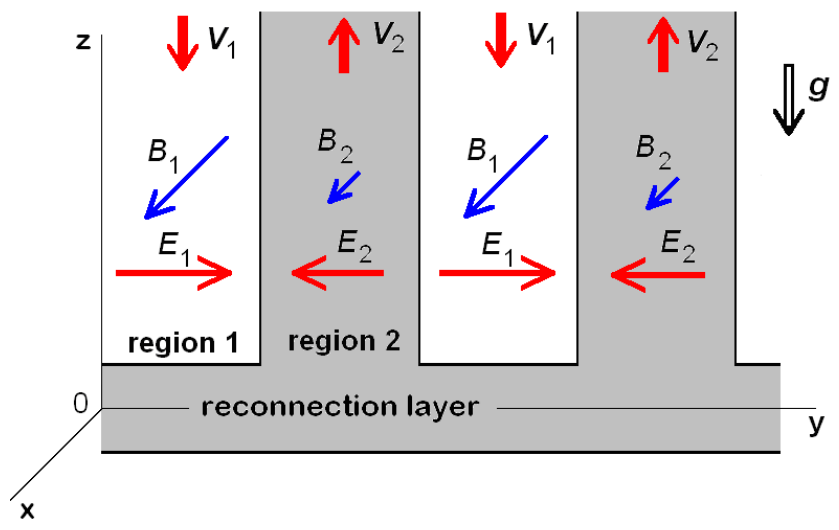

Fig. 6. Schematic of resulting steady-state Rayleigh-Bénard convection. Region 1 is related to plasma flows toward the reconnection layer, while region 2 is related to plasma flows out of this layer. Magnetic fields $\boldsymbol{B}_{1}$ and $\boldsymbol{B}_{2}$ are normal to the plane of the figure, $\boldsymbol{g}$ is an effective gravitational acceleration, $\boldsymbol{E}_{1}$ and $\boldsymbol{E}_{2}$ are the polarization electric fields, and $\boldsymbol{V}_{1}$ and $\boldsymbol{V}_{2}$ are the $\boldsymbol{E} \times \boldsymbol{B}$ drift velocities in the regions 1 and 2 , respectively. The convection is shown only in the region above the reconnection layer; below the reconnection layer, the magnetic fields have the opposite directions (not shown).

1. We found that in a realistic model of interacting magnetic fields involving a force normal to the reconnection layer, the reconnection layer with heated plasma is unstable against interchange perturbations.

2. The interchange instability results in formation of tongues of heated plasma leaving the reconnection layer not through its narrow ends but through its wide surface, which provides fast removal of plasma from the reconnection layer and, as a result, leads to fast reconnection. This instability can explain bidirectional (toward and out from the reconnection layer) plasma fluxes, observed in the vicinity of the reconnection layer (e.g., McFadden et al., 2008). Note that Pritchett and Coroniti (2010) studied the instability not exactly on the reconnection layer but on the dipolarization fronts, which are generated by magnetic reconnection in the magnetotail and propagate ahead of the reconnection layer (e.g., Guzdar et al., 2010; Lapenta and Bettarini, 2011). The instability is caused by the inertial force normal to the reconnection layer and dipolarization fronts, and appearing on these fronts (and, possibly, also in the reconnection layer if it moves with acceleration).

3. The proposed mechanism provides fast reconnection rate of interacting magnetic fields, which does not depend on the length of the reconnection layer. 


\section{Turbulent reconnection and internal plasmoid instability}

\subsection{Introduction}

Magnetic reconnection in turbulent plasma is significantly different from classical reconnection. Measurements at different distances from the Earth showed strong variability of solar wind/interplanetary magnetic field parameters (Coleman, 1968; Belcher and Davis, 1971; Goldstein and Roberts, 1999; Goldstein, 2001; and others). Strong variability of plasma and magnetic field parameters in the reconnection region makes the traditional assumption of a laminar equilibrium inappropriate; in this case, reconnection events may be randomly scattered in space and time. The term "turbulent reconnection" (Matthaeus et al., 1984; Goldstein et al., 1986; Matthaeus and Lamkin, 1986; Lazarian and Vishniac, 1999, and others) applies to such a situation.

Shibata and Tanuma (2001), Daughton et al. (2009), Cassak et al. (2009), among others, additionally found that the reconnection layer also is unstable to small-scale perturbations, which leads to formation of "a chain of plasmoids" (so-called "secondary islands"). A possible cause for formation of these islands can be the common tearing instability. The rate of formation of such islands is found to be very fast (larger than the Alfvénic rate), and their formation was suggested to be "a generic feature of reconnecting systems" (Loureiro et al., 2011).

Turbulent reconnection due to both external turbulence and internal instability leads to multiple reconnection of magnetic field lines and "chopping" the reconnection layer into smaller lengths. Matthaeus and Lamkin (1986), Lazarian and Vishniac (1999, 2000), and Shibata and Tanuma (2001), among others, suggested that this process could increase the reconnection rate due to decreasing the effective length of the reconnection layer or by causing an increase in local, anomalous resistivity associated with these small-scale plasma islands. However, since turbulent reconnection is associated with the formation of "plasma islands" in the reconnection region, a piling-up of these islands around the reconnection layer could also prevent inflowing new plasma and magnetic field into the reconnection layer, which would reduce the reconnection rate. Indeed, results of recent simulations (e.g., Shepherd and Cassak, 2010) showed that the formation of the plasma/magnetic islands results only in a relatively insignificant increase in the reconnection rate and, therefore, these authors suggested that the formation of such islands is unlikely to resolve the problem of fast reconnection. These results are related, however, to so-called "secondary islands", produced by an internal instability of the reconnection layer, which in more detail is considered below.

Another possible mechanism for the formation of the plasma islands in the vicinity of the reconnection layer may be related to the disruption of large-amplitude interchange perturbations (e.g., Nakamura et al., 2002; Guzdar et al.,

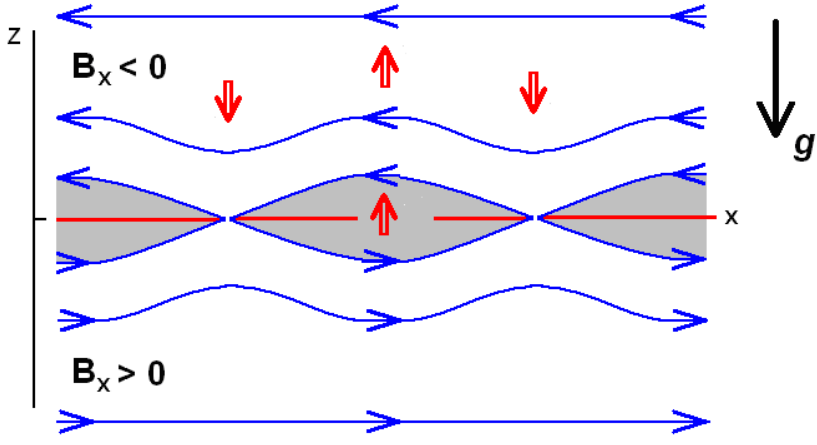

Fig. 7. Formation of plasma islands as a result of magnetic reconnection in combination with turbulent magnetic fields or interchange perturbations oriented at some angle to the magnetic field. The appearing islands of heated plasma move upward against the gravitational acceleration, $g$. The blue lines show the magnetic field; the red arrows show upward motion of the plasma bubbles and downward flow of the surrounding plasma.

2010; and references therein) in their non-linear stage. The variety of mechanisms responsible for the formation of the plasma islands leads to variety in their shapes and sizes.

A simple model for formation of the plasma islands in the case of turbulent reconnection is shown in Fig. 7. Such islands, as mentioned above, may appear due to turbulence in the surrounding plasma or due to an internal instability of the reconnection layer. As a result of turbulent reconnection, multiple reconnection can form multiple plasma islands that are surrounded by closed (force-free) magnetic fields, as shown in this figure.

The sizes of resulting plasma islands are derived in this case primarily by the wave length of external turbulence or internal perturbations along the ambient magnetic field, which lead to the multiple reconnections and the formation of plasma islands. However, the size of these islands can be lesser than predicted because of the magnetic tension force $(\boldsymbol{B} \cdot \nabla \boldsymbol{B}) / 4 \pi \approx B^{2} / 4 \pi R_{\mathrm{c}}$, where $R_{\mathrm{c}}$ is the radius of magnetic field curvature. This force is at its maximum on the sharp ends of the plasma islands where $R_{\mathrm{c}}$ is small, which decreases the length of the plasma islands.

In the recent years, it was also found (Loureiro et al., 2007; Lapenta, 2008; Daughton et al., 2009; Samtaney et al., 2009; Shepherd and Cassak 2010; Lapenta and Bettarini, 2011; and many others) that the current sheets in the reconnection layer in the process of magnetic reconnection are unstable against the formation 435 of plasma/magnetic islands, known also as the plasmoids and "secondary islands". In the non-linear regime, these plasmoids are found to grow "faster than they are ejected and completely disrupt the reconnection layer" (Samtaney et al., 2009), which may significantly effect the reconnection rate.

The processes related to the generation and removing of these plasma islands from the reconnection layer, as well 
as their effect on the reconnection rate, are not totally clear yet. Shepherd and Cassak (2010) found that in the case of a relatively small amount of such islands, the reconnection rate can increase and be faster "than Sweet-Parker but significantly slower than Hall" reconnection rate (Shepherd and Cassak, 2010). In the case of a large number of these islands, their effect on reconnection rate can be, probably, even more significant since the reconnection rate is dependent on the rate of removal of these islands from the system. Particularly, Samtaney et al. (2009) stated that "for sufficiently large systems, plasmoid-dominated current layers are inevitable, and they may be key to attaining fast reconnection, both in collisional and collisionless systems. Plasmoid formation and magnetic reconnection are thus inextricably linked, and further progress in understanding reconnection in realistic systems necessarily requires a theory that takes the plasmoid dynamics into account".

Although the mechanism of the formation of plasma islands and their role in reconnection are not totally clear yet, we will show that turbulent reconnection, associated with the formation of plasma islands, in some circumstances can lead to a significant increase in the reconnection rate when the force normal to the reconnection layer is included in the model. In this case, the plasma islands may be quickly expelled from the reconnection layer due to resulting effective buoyancy force. The physics of expelling these islands is similar to the R-T instability but is related to isolated plasma formations in the magnetic field in the presence of a gravitational (or pseudo-gravitational) force.

\subsection{Removal of plasma islands from reconnection region}

Above we discussed some possible mechanisms for the formation of plasma bubbles which include external turbulence, internal instabilities (such as the tearing and kink instabilities) in the reconnection layer, and the formation of detached plasma bubbles at non-linear stage of the interchange instability (Nakamura et al., 2002; Guzdar et al., 2010). Our purpose is to discuss the effect of such plasma islands on the reconnection rate. As mentioned above, plasma islands, pilingup in the reconnection region and its vicinity, can reduce the reconnection rate in the traditional reconnection models. Here we show that the force normal to the reconnection layer can significantly increase the velocity at which the plasma islands, pushed away from the reconnection layer by the buoyancy force or its analogue, leave the reconnection layer and its vicinity, which increases the reconnection rate.

It is well known that a cloud (bubble) of hot plasma in an inhomogeneous magnetic field is expelled toward the weak magnetic field (against $\nabla B$ ). This fact is explained as a result of diamagnetism of this plasma cloud or $\nabla B$ drift of its particles so that the arising polarization electric field expels the plasma cloud toward the weaker magnetic field. In the case of Earth's magnetosphere, the resulting polarization electric
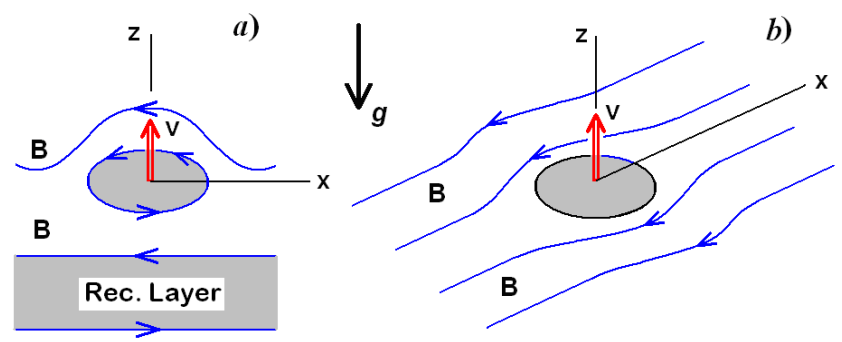

Fig. 8. A schematic showing a detached quasi-spherical bubble, filled in with hot plasma and surrounded by closed magnetic field lines (shown by blue lines) as a result of magnetic reconnection; the closed magnetic field supports the bubble in equilibrium. $\boldsymbol{B}$ is the magnetic field, $\boldsymbol{g}$ is the quasi-gravitational acceleration directed parallel to the z-axis normally to the reconnection layer. The ambient magnetic field is assumed to decrease along the $\mathrm{z}$-axis. The hot plasma bubble is expelled toward the weaker magnetic field (along the z-axis) due to the effective buoyancy force and moves upward at the velocity $\boldsymbol{V}$. The left panel shows the motion of the bubble in the $\mathrm{x}-\mathrm{z}$ plane. The right panel shows motion of the bubble in 3-D space; the bubble moves upward in between the ambient magnetic field lines.

field removes the plasma cloud away from the Earth; note that it is the same polarization electric field that is responsible for developing the interchange instability on the plasma sheet outer boundary (e.g., Lyatsky and Maltsev, 1983; Maltsev, 1986). A similar situation occurs in the case of hot plasma islands, generated in the reconnection layer and expelled due to turbulence or other mechanisms in the ambient plasma (e.g., Guzdar et al., 2010). These plasma islands are polarized in the external inhomogeneous magnetic field and due to $\boldsymbol{E} \times \boldsymbol{B}$, drift of hot particles in the polarization electric field, $E$, and the ambient magnetic field, $B$, and can continue to move away from the reconnection layer against the effective gravitational acceleration, $g$. The motion of these islands can also be described as the motion under the diamagnetic force $-\mu n \nabla \boldsymbol{B}$, where $\mu$ is the magnetic moment of the island particles, and $n$ is their number density.

The velocity of the plasma bubbles depends on properties of these bubbles and surrounded plasma and, among the other parameters, on their geometry. For simplicity, we discuss here two simple cases when the plasma bubbles have a quasi-spherical form and when they are elongated along the magnetic field. The first case is shown in Fig. 8.

In a pseudo-gravitational field, associated with a force normal to the interface between two magnetic fields, the isolated plasma islands can move upward or downward dependently on the direction of the acting force. Since the plasma temperature within plasma bubbles is usually larger than that in the surrounding plasma, they move against the gravitational or pseudo-gravitational force as shown in Fig. 8. Assuming that the bubbles, detached from the reconnection layer, contain heated plasma and a reduced magnetic field, the plasma pressure in the bubble $p_{1}$ should be much higher than that in the 
ambient plasma, i.e., $p_{1} \gg p_{2}$, while the magnetic pressure in the bubble $B_{1}^{2} / 8 \pi \ll B_{2}^{2} / 8 \pi$, where index 2 is related to the region outside the bubble. Then in a quasi-stationary case from the balance of total (plasma plus magnetic field) pressures, we have $p_{1} \approx B_{2}^{2} / 8 \pi$. For simplicity, we neglected here the magnetic tension force. Then the magnetic field outside the totally diamagnetic bubble of a quasi-spherical shape near its boundary is $B_{2} \approx 2 B_{0}$, where $B_{0}$ is the ambient magnetic field. As a result, we have

$p_{1} \approx 2 B_{0}^{2} / 8 \pi \gg p_{0}$.

Note that the magnetic tension force increases plasma pressure $p_{1}$ even more within the bubble.

The differences in plasma densities, temperatures, and pressures inside and outside the bubble lead to vertical motion of the bubble in both real and effective "gravitational" fields. Since magnetic tension does not affect the vertical motion of isolated plasma bubbles surrounded by closed magnetic field lines (as they move in between the ambient magnetic field lines as shown in Fig. 8b), these bubbles may move relatively freely in the effective gravitational field until their velocity is small enough. While moving upward, they are rapidly accelerated to a terminal velocity, $V_{\text {term }}^{\uparrow}$, which is defined from the balance between the buoyant force and a drag force which is a function of velocity. The exact value of the termination velocity of such bubbles in not well known (e.g., Longcope et al., 1996; Emonet and Moreno-Insertis, 1998), but it is reasonable to suggest that their velocity is unlikely to be higher than the Alfvén velocity since in super-Alfvénic regime the energy loss of the bubbles on wave generation become very high. Therefore, as a first approximation, we can suggest that, by neglecting viscosity of plasma, the terminal velocity of the plasma bubbles in a real or effective gravitational field is close to the Alfvén velocity in the ambient plasma, which provides the fast removal of such plasma bubbles from the reconnection layer and its vicinity, and may support the fast reconnection rate.

Now we consider the case of bubbles of elliptic shape, extended at some angle with respect to the magnetic field. In this case, their motion away from the reconnection layer can deform the surrounding magnetic field, which reduces the island velocity. However, even in this case, the effective buoyant force can rotate these islands to make them to be oriented along the magnetic field (see, e.g., Uchida and Sakurai, 1977). When plasma islands are oriented along the magnetic field, they move in between the ambient magnetic field lines without their significant violation; the size of plasma islands along the magnetic field in this case is not essential. This case, which can be described as the motion of a plasma roll, is shown schematically in Fig. 9.

By neglecting viscosity, the terminal velocity, $V_{\text {term }}^{\uparrow}$, of a flux tube, filled with heated plasma and moving in the ambient magnetic field, may be estimated by equaling the drift currents within this tube to the currents at its boundary

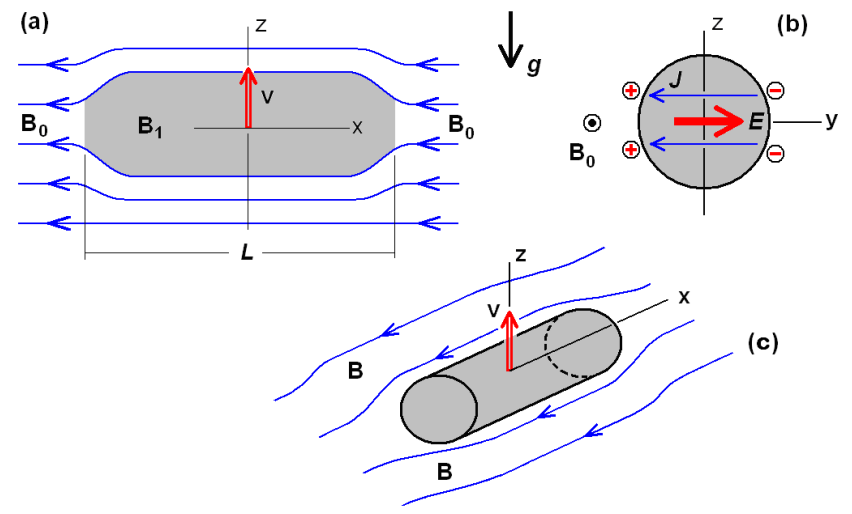

Fig. 9. Motion of a plasma roll extended along the ambient magnetic field $\boldsymbol{B}_{0}$ shown by blue lines. $\boldsymbol{B}_{1}$ is the magnetic field within this roll, $L$ is its length, $\boldsymbol{g}$ is the effective gravitational acceleration, and $\boldsymbol{V}$ is the $\boldsymbol{E} \times \boldsymbol{B}$ drift velocity. The ambient magnetic field is assumed to decrease along the z-axis, which is normal to the reconnection layer (not shown). The hot plasma roll is expelled toward the weaker magnetic field (along the $\mathrm{z}$-axis) due to the effective buoyancy force and moves upward at the velocity $V$. (a) shows the motion of the roll in the X-z plane. (b) shows the drift currents $\boldsymbol{J}$ (blue lines) and polarization electric field $\boldsymbol{E}$ in the cross-section of the magnetic role; the ambient magnetic field $\boldsymbol{B}_{0}$ here is directed across the figure plane. (c) shows motion of the roll in 3-D space; the roll moves upward in between the ambient magnetic field lines.

and/or ends. Let us consider a case, when the ends of the magnetic flux tube with hot plasma are immersed in the conducting layer, which may be related to the solar photosphere or Earth's ionosphere (Maltsev, 1986; Longcope et al., 1996; Lyatsky et al., 2010). In solar photosphere and corona, such a situation appears when a fresh magnetic field goes out from solar interior and moves upward to the solar corona where there is an ambient oppositely directed magnetic field (see, e.g., Longcope et al., 1996) that can result in the magnetic reconnection.

The polarization electric field, generated within the flux tube due to the drift of charged particles in the gravitational and magnetic fields, leads to the generation of the Alfvén waves, propagated towards the ends of the tube. The ion drift velocity across the flux tube is derived as

$V_{1,2}=c\left|\frac{m \boldsymbol{g} \times \boldsymbol{B}_{1,2}}{e B_{1,2}^{2}}\right|=c \frac{m g}{e B_{1,2}}$,

where indices 1 and 2 are related to the regions inside and outside the tube, respectively. Electrons drift in the opposite direction, but due to their small mass, their drift may be neglected. Then the maximal density of currents, entering the boundary of the flux tube and integrated over its half-length, $L$, is

$J_{y}=e\left(n_{1} V_{y 1}-n_{2} V_{y 2}\right) L$,

so that the current $J_{y}$ has the dimension of a surface current. These currents lead to the polarization electric field 


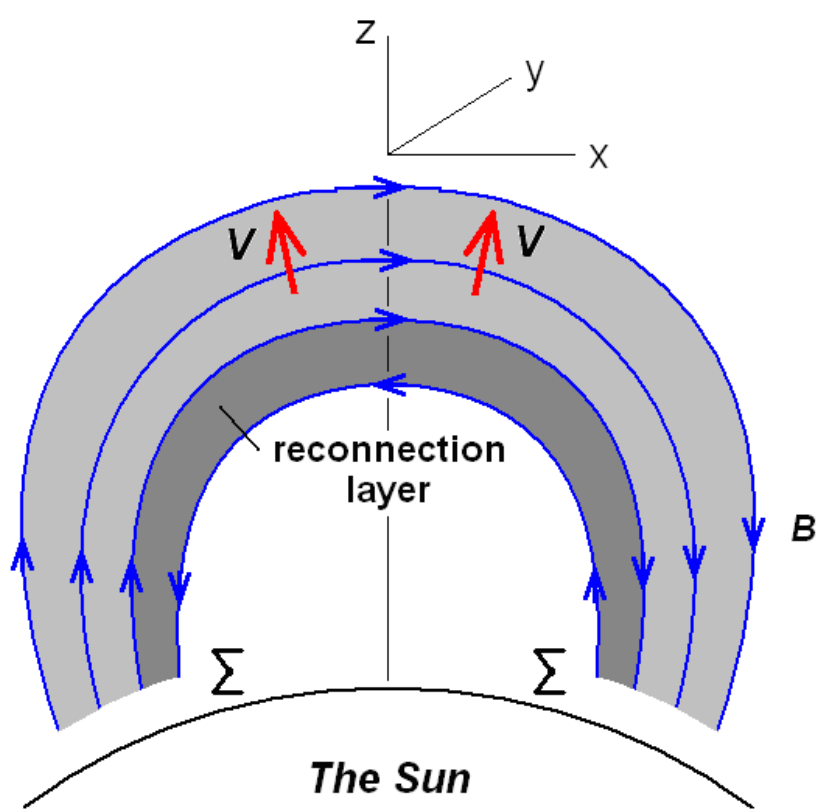

Fig. 10. A schematic showing upward motion of a magnetic flux tube, filled with hot plasma, when the ends of this tube are immersed in a conducting layer near the solar photosphere. The flux tube, filled with plasma heated in the reconnection layer, is rising upward at a velocity $\boldsymbol{V} ; \Sigma$ is the height-integrated conductivity of the solar photosphere. The blue lines show the magnetic field lines.

across the tube and generation of two Alfvén waves propagated along the magnetic field in both directions (e.g., Lyatsky et al., 2010). These waves transport the electric field and the field-aligned currents to the edges of the tube. The magnitude of the polarization currents in each Alfvén wave, integrated across its front, may be derived as (Lyatsky and Maltsev, 1983; Lyatsky et al., 2010)

$J_{\mathrm{pol}}=2 \Sigma E_{y}$,

where $E_{y}$ is the polarization electric field, the factor 2 is due to the fact that the polarization currents are flowing both inside and outside the cross-section of the flux tube through the conductive layer in the solar photosphere (for the tube of a circular cross-section, the currents inside and outside its cross-section are equal; Lyatsky et al., 2010). Since the total currents are continuous, $J_{y}$ in Eq. (15) is equal to $J_{\text {pol }}$ in Eq. (16). Equating Eqs. (15) and (16) yields

$e\left(n_{1} V_{y 1}-n_{2} V_{y 2}\right) L=2 \Sigma E_{y}$.

From this equation, we obtain the electric field across the tube

$E_{y}=\frac{e\left(n_{1} V_{y 1}-n_{2} V_{y 2}\right) L}{2 \Sigma}$.

Accounting for Eq. (18) and assuming $n_{1} V_{y 1} \gg n_{2} V_{y 2}$ gives then the following simple estimate for the vertical terminal velocity of the flux tube:

$$
V_{z}^{\uparrow}=c \frac{E_{y}}{B_{1}}=c \frac{e\left(n_{1} V_{z 1}-n_{2} V_{z 2}\right) L}{2 B_{1} \Sigma}=\frac{g L}{2 V_{\mathrm{A} 1}} \frac{\Sigma_{\mathrm{A} 1}}{\Sigma},
$$

where $\Sigma_{\mathrm{A} 1}$ is the Alfvén conductance $\Sigma_{\mathrm{A} 1}=c^{2} / 4 \pi V_{\mathrm{A} 1}$. Note that the upward velocity of the tube is proportional to its length; it means that this velocity increases with the size of the system and the reconnection region, which in space physics can be very large.

Thus, in this case a force normal to the reconnection layer can increase the velocity at which the plasma islands, filled with hot plasma, are expelled away from the reconnection layer, which can significantly increase the reconnection rate. The plasma islands, generated within and in the vicinity of the reconnection layer, are removed away from the reconnection layer by two ways: (1) together with flows of outgoing plasma streams as a part of the Rayleigh-Bénard convection (as shown in the Sect. 3 and Fig. 6), and (2) independently due to the effective buoyancy force that expels these plasma islands away from the reconnection layer. The plasma islands extended at a significant angle with respect to the ambient magnetic field can move mainly with outgoing plasma streams as a part of the Rayleigh-Bénard convection; the plasma islands of a quasi-spherical shape or extended along the ambient magnetic field can also move independently due to the effective buoyancy force, which expels the islands away from the reconnection layer. Note, however, that even in this case, the effective buoyant force can rotate these islands to make them be oriented along to the magnetic field (see, e.g., Uchida and Sakurai, 1977).

\section{Summary}

We present here the results of a study of magnetic reconnection that involves a force normal to the reconnection layer. In the presence of such force, the reconnection layer can become unstable to interchange disturbances. The interchange instability results in formation of tongues of heated plasma that leaves the reconnection layer through its wide surface rather than through its narrow ends, as is the case in traditional reconnection models. This plasma flow out of the reconnection layer facilitates the removal of plasma from the layer that leads to fast reconnection. The interchange instability also explains bidirectional streaming of plasma toward and away from the reconnection layer that is observed frequently in the vicinity of reconnection layers. The force normal to the reconnection layer also facilitates the removal of plasma islands appearing in the reconnection layer during turbulent reconnection; in this case, these islands are removed from the reconnection layer by the "buoyancy force" appearing due to the polarization electric field generated at their boundaries. Thus, a force normal to the reconnection layer can play the role of a catalyst that can significantly accelerate the reconnection rate. 
The same as in the Sweet-Parker and Petschek mechanisms, the reconnection rate $V_{\mathrm{RR}}$, given by Eq. (6), shows the maximum possible reconnection rate (a "channel capacity"), which is equal to the maximum possible rate of the removal of piled-up plasma out from the reconnection layer (see, e.g., Parker, 1957; Petschek, 1964; Priest and Forbes, 2000; and reference therein). The reconnection rate depends predominantly on the Alfvén velocity in inflowing plasma as well as the mechanism of plasma removal from the reconnection layer, which includes the geometry of the problem. The term "slow reconnection rate" is conventionally related to the case when the reconnection rate $V_{\mathrm{RR}} \ll V_{\mathrm{A}}$, while "fast reconnection rate" is related to the case when $V_{\mathrm{RR}} \approx V_{\mathrm{A}}$. In the case when the density ratio $\rho_{2} / \rho_{1}$ is not very significantly different from 1, Eq. (12) for reconnection rate can be rewritten as

$V_{\mathrm{RR}} \approx V_{\mathrm{A}}$,

which shows that the proposed mechanism is able to provide fast reconnection. The real reconnection rate cannot exceed the magnitude of $V_{\mathrm{RR}}$ but can be less than that. If the flux of plasma entering the reconnection layer exceeds the maximum possible flux of outflowing plasma, a superfluous flux of the plasma with the magnetic field will go about the reconnection layer. A similar situation takes place possibly in the case of magnetic reconnection on Earth's magnetopause, where the reconnection occurs only for about 10 per cent of incident magnetic flux, even when the interplanetary magnetic field is anti-parallel to Earth's magnetic field (e.g., Yamada et al., 2010).

As mentioned above, fast reconnection in the proposed mechanism is a result of plasma removal from the reconnection layer, not along the narrow reconnection layer (as in the Sweet-Parker mechanism) but through its wide boundary, which is a result of the combined effect of reconnection and interchange instability. Thus, the interchange instability of heated plasma in the reconnection region can provide and support fast reconnection. Though the interchange instability in many cases has a low or even no threshold, its development can be effected by local turbulence, velocity shear, and other factors; this instability, therefore, should have sufficiently high growth rate to overcome these factors in order to support the fast reconnection rate.

As an example, we estimate here a growth rate of the gravitational interchange instability which can develop on reconnection layers in the solar corona. The characteristic time of gravitational interchange instability is derived from Eq. (6). Let us consider the case when the $\boldsymbol{k}$-vector of interchange perturbations is directed across the magnetic field, and the ratio $j \rho_{1}-\rho_{2} j /\left(\rho_{1+} \rho_{2}\right) \geq 1$ (the sign of $\left(\rho_{1}-\rho_{2}\right)$ in this case is not important: the lighter plasma of the layer tends to move upward while the heavier one downward). In this case, the growth rate can be written in a simple form

$\gamma^{2} \approx k g$ where $k$ is the wave number along the reconnection layer, and $g$ is the gravitational acceleration. In this case, the characteristic time of the interchange instability, $\tau=1 / \gamma$, can be written as follows:

$\tau=\frac{1}{\gamma} \approx\left(\frac{\lambda}{2 \pi g}\right)^{1 / 2}$

where $\lambda=2 \pi / k$ is the wave length along the reconnection layer. Using this formula, we can estimate the characteristic time of the instability in the solar corona, where reconnection of oppositely directed magnetic fields is thought to be a rather common phenomenon (e.g., Yamada et al., 2010; Crooker et al., 2012).

As an example, we estimate the characteristic time of this interchange instability in the inner corona at the altitude of about $2 R_{\mathrm{S}}$ where $R_{\mathrm{S}}$ is the solar radius. The gravitational acceleration at the solar surface is about $274 \mathrm{~m} \mathrm{~s}^{-2}$, while at the radial distance $r=2 R_{\mathrm{S}}$ it is $\sim 68 \mathrm{~m} \mathrm{~s}^{-2}$. In this case, from Eq. (22) we can obtain

$\tau(s) \approx 2.4 \times 10^{2}(\lambda(\mathrm{deg}))^{1 / 2}$,

where the characteristic time, $\tau$, is measured in seconds, and the wavelength, $\lambda$, in the degrees along the latitude or longitude near the equatorial plane (one degree at the radial distance $r=2 R_{\mathrm{S}}$ corresponds to about $24 \times 10^{3} \mathrm{~km}$ ). For reasonable possible magnitudes of $\lambda=(0.1-10)^{\circ}$, we obtain $\tau \approx$ (77-770) s. At the radial distance $r=5 R_{\mathrm{S}}$, the characteristic time of the instability for the same interval of $\lambda$ increases by the factor of about 4 . These characteristic times are relatively low, especially by accounting for the huge sizes (thousands of kilometers) of the instability, and they decrease even more for shorter wavelengths. These characteristic times of the instability also are less than the typical periods (several hours) of strong turbulence in the solar wind, which is thought to be generated in the solar corona (e.g., Goldstein, 2001).

The estimates above show that the interchange instability indeed may play a significant role in the reconnection in the solar corona, which is consistent with the results of simulations by Uchida and Sakurai (1977) (see also Kahler et al., 1980; McAllister et al. 1994; Uchida, 1996; and references therein), though the mechanism, as proposed by these authors, is different from that proposed in our paper. They suggested that the development of this instability on the boundary of the reconnection layer results in the formation "of thin sheets of plasma invaded into the region of opposite polarity", which enhances the reconnection rate. Although their mechanism does not resolve the fast removal of piled-up plasma out off the reconnection layer, nevertheless, it may play an additional role in increasing the reconnection rate.

An important consequence of interchange instability is the formation of bidirectional (toward and out of the reconnection layer) plasma fluxes. Such fluxes are frequently observed in the vicinity of the reconnection region. Bidirectional plasma flows were observed, for example, near dayside 
magnetopause with the Themis spacecraft (e.g., McFadden et al., 2008; Liu et al., 2008; the first paper is related to a case of the negative Interplanetary Magnetic Field (IMF), while the second paper is related to a series of flux transfer events). However, correct identification of interchange perturbations at Earth's magnetopause is difficult due to the large velocity shear near the magnetopause, which can be the cause for the generation of another (e.g., Kelvin-Helmholtz) instability. Note that bidirectional fluxes of plasma were also observed in Earth's magnetotail during the formation of socalled "dipolarization fronts" after magnetic reconnection in the magnetotail. These fluxes are also suggested to be a result of interchange instability, though the cause for this instability may be another: This instability may be generated not at the boundary of the reconnection layer but rather at the dipolarization fronts, generated after magnetic reconnection in the magnetotail (e.g., Pritchett and Coroniti, 2000, 2010; Guzdar et al., 2010; Lapenta and Bettarini, 2011; and references therein).

Thus, the presented study shows that there are a wide variety of different mechanisms responsible for magnetic reconnection, which provides different ways for the removal of plasma piled-up in the reconnection layer, which results in fast reconnection rates.

Acknowledgements. The authors are grateful to John Dorelli for useful discussions. This research was supported, in part, by the NASA Cluster Project at the Goddard Space Flight Center.

Edited by: T. Intrator

Reviewed by: two anonymous referees

\section{References}

Belcher, J. W. and Davis Jr., L.: Large-amplitude Alfvén waves in the interplanetary medium, 2, J. Geophys. Res., 76, 3534-3563, 1971.

Birn, J., Drake, J. F., Shay, M. A., Rogers, B. N., Denton, R. E., Hesse, M., Kuznetsova, M., Ma, Z. W., Bhattacharjee, A., Otto, A., and Pritchett, P. L.: Geospace Environmental Modeling (GEM) Magnetic Reconnection Challenge, J. Geophys. Res., 106, 3715-3719, 2001.

Biskamp, D.: Magnetic Reconnection Via Current Sheets, Phys. Fluids, 29, 1520-1531, 1986.

Bret, A.: Intuitive calculation of the relativistic Rayleigh-Taylor instability linear growth rate, Laser Particle Beams, 29, 255-257, 2011.

Cassak, P. A. and Shay, M. A.: Scaling of asymmetric Hall magnetic reconnection, Geophys. Res. Lett., 35, L19102, doi:10.1029/2008GL035268, 2008.

Cassak, P. A., Shay, M. A., and Drake, J. F.: Catastrophe Model for Fast Magnetic Reconnection Onset, Phys. Rev. Lett., 95, 2350021-4, doi:10.1103/PhysRevLett.95.235002, 2005.

Cassak, P. A., Shay, M. A., and Drake, J. F.: Scaling of SweetParker reconnection with secondary islands, Phys. Plasma, 16, 120702, doi:10.1063/1.3274462, 2009.
Chandrasekhar, S.: Hydrodynamic and Hydromagnetic Stability, Oxford, Clarendon Press, 1961.

Coleman, P. J.: Turbulence, viscosity, and dissipation in the solar wind plasma, Astrophys. J., 153, 371, doi:10.1086/149674, 1968.

Crooker, N. U., Antiochos, S. K., Zhao, X., and Neugebauer, M.: Global network of slow solar wind, J. Geophys. Res., 117, A04104, doi:10.1029/2011JA017236, 2012.

Daughton, W., Scudder, J., and Karimabadi, H.: Fully kinetic simulations of undriven magnetic reconnection with open boundary conditions, Phys. Plasmas, 13, 072101, doi:10.1063/1.2218817, 2006.

Daughton, W., Roytershteyn, V., Albright, B. J., Karimabadi, H., Yin, L., and Bowers, K. J.: Transition from collisional to kinetic regimes in large-scale reconnection layers, Phys. Rev. Lett., 103, 065004, doi:10.1103/PhysRevLett.103.065004, 2009.

Dorelli, J. C. and Birn, J.: Whistler-mediated magnetic reconnection in large systems: Magnetic flux pileup and the formation of thin current sheets, J. Geophys. Res., 108, 1133, doi:10.1029/2001JA009180, 2003.

Emonet, T. and Moreno-Insertis, F.: The Physics of Twisted Magnetic Tubes Rising in a Stratified Medium: Two-dimensional Results, Astrophys. J., 492, 804, doi:10.1086/305074, 1998.

Frank-Kamenetsky, D. A.: Lectures on plasma physics, Atomizdat, Moscow, 1964.

Goldstein, M. L.: Major unsolved problems in space plasma physics, Astrophys. Space Sci., 277, 349-369, 2001.

Goldstein, M. L. and Roberts, D. A.: Magnetohydrodynamic turbulence in the solar wind, Phys. Plasmas, 6, 349-369, 1999.

Goldstein, M. L., Matthaeus, W. H., and Ambrosiano, J. J.: Acceleration of charged particles in magnetic reconnection: Solar flares, the magnetosphere, and solar wind, Geophys. Res. Lett., 13, 205-208, 1986.

Gratton, F. T., Farrugia, C. J., and Cowley, S. W. H.: Is the magnetopause Rayleigh-Taylor unstable sometimes?, J. Geophys. Res., 101, 4929, doi:10.1029/95JA03064, 1996.

Guzdar, P. N., Hassam, A. B., Swisdak, M., and Sitnov, M. I.: A simple MHD model for the formation of multiple dipolarization fronts, Geophys. Res. Lett., 37, L20102, doi:10.1029/2010GL045017, 2010.

Huang, Y.-M., Bhattacharjee, A., and Sullivan, B. P.: Onset of fast reconnection in Hall magnetohydrodynamics mediated by the plasmoid instability, Phys. Plasmas, 18, 072109 , doi:10.1063/1.3606363, 2011.

Huba, J. D.: Finite Larmor radius magnetohydrodynamics of the Rayleigh-Taylor instability, Phys. Plasmas, 3, 2523-2532, 1996.

Jun, B.-I., Norman, M. L., and Stone, J. M.: A Numerical Study of Rayleigh-Taylor Instability in Magnetic Fluids, Astrophys. J., 453, 332-349, 1995.

Kahler, S., Spicer, D., Uchida, Y., and Zirin, H.: Primary energy release, in: "Solar Flares", edited by: Sturrock, P., Assoc. Univ. Press, Boulder, CO, 1980, 83-116, 1980.

Kulsrud, R. M.: Magnetic reconnection: Sweet-Parker versus Petschek, Earth Planet. Space, 53, 417-422, 2001.

Lapenta, G.: Self-feeding turbulent magnetic reconnection on macroscopic scales, Phys. Rev. Lett., 100, 235001, doi:10.1103/PhysRevLett.100.235001, 2008.

Lapenta, G. and Bettarini, L.: Self-consistent seeding of the interchange instability in dipolarization fronts, Geophys. Res. Lett., 38, L11102, doi:10.1029/2011GL047742, 2011. 
Lazarian, A. and Vishniac, E.: Reconnection in a weakly stochastic field, Astrophys. J., 517, 700, doi:10.1086/307233, 1999.

Lazarian, A. and Vishniac, E.: Fast reconnection of magnetic fields in turbulent fluids, RevMexAA (Serie de Conferencias), 9, 55$62,2000$.

Liberatore, S. and Bouquet, S.: Analytical modeling of magnetic Rayleigh-Taylor instabilities in compressible fluids, Phys. Fluids, 20, 116101, doi:10.1063/1.3025832, 2008.

Livescu, D.: Compressibility effects on the Rayleigh-Taylor instability growth between immiscible fluids, Phys. Fluids, 16, 118125, 2004.

Liu, J., Angelopoulos, V., Sibeck, D., Phan, T., McFadden, J., Glassmeier, K. H., Auster, U., and Pu, Z.-Y.: THEMIS Observations of the Dayside Traveling Compression Region and Flows Surrounding Flux Transfer Events, Geophys. Res. Lett., 35, L17S07, doi:10.1029/2008GL033673, 2008.

Longcope, D. W., Fisher, G. H., and Arendt, S.: The Evolution and Fragmentation of Rising Magnetic Flux Tubes, Astrophys. J., 464, 999-1011, doi:10.1086/177387, 1996.

Loureiro, N. F., Schekochihin, A. A., and Cowley, S. C.: Instability of current sheets and formation of plasmoid chains, Phys. Plasmas, 14, 100703, doi:10.1063/1.2783986, 2007.

Lyatsky, W. B. and Goldstein, M. L.: A Simple Model of Fast Magnetic Reconnection, AGU, Fall Meeting 2010, abstract \# SM42A-05, 2010.

Lyatsky, W. B. and Maltsev, Y. P.: Magnetosphere-Ionosphere Interaction, "Nauka", Moscow, 1983.

Lyatsky, W. B. and Sibeck, D.: Central plasma sheet disruption and the formation of dayside poleward moving auroral events, J. Geophys. Res., 102, 17625-17630, 1997.

Lyatsky, W., Khazanov, G. V., and Slavin, J. A.: Saturation effect in electric field transmitted into magnetosphere, J. Geophys. Res., 115, A08221, doi:10.1029/2009JA015091, 2010.

Maltsev, Y. P.: Perturbations in the Magnetosphere-Ionosphere System, Academy of Science, Polar Geophysical Institute, Apatity, Russia, 1986.

Matthaeus, W. H. and Lamkin, S. L.: Turbulent magnetic reconnection, Phys. Fluids, 29, 2513-2534, 1986.

Matthaeus, W. H., Ambrosiano, J. J., and Goldstein, M. L.: Particle acceleration by turbulent magnetohydrodynamic reconnection, Phys. Rev. Lett., 53, 1449-1452, 1984.

McAllister, A. H., Uchida, Y., Khan, J. I., and Shibata, K.: Coronal magnetic fields parallel to magnetic polarity inversion lines, in: X-ray Solar Physics from Yohkoh, edited by: Uchida, Y., Watanabe, T., Shibata, K., and Hudson, H. S., Universal Acad. Press, Tokyo, 189 pp., 1994.

McFadden, J. P., Carlson, C. W., Larson, D., Bonnell, J., Mozer, F., Angelopoulos, V., Glassmeier, K.-H., and Auster, U.: THEMIS ESA First Science Results and Performance Issues, Space Sci. Rev., 141, 477-508, 2008.

Nakamura, M. S., Matsumoto, H., and Fujimoto, M.: Interchange instability at the leading part of reconnection jets, Geophys. Res. Lett., 29, 1247, doi:10.1029/2001GL013780, 2002.
Parker, E. N.: Sweet's mechanism for merging magnetic fields in conducting fluids, J. Geophys. Res., 62, 509-520, 1957.

Petschek, H. E.: Physics of solar flares, edited by: Hess, W. N., NASA, SP-50, Washington, DC, 425-439, 1964.

Piriz, A. R., Cortázar, O. D., López Cela, J. J., and Tahir, N. A.: The Rayleigh-Taylor instability, Am. J. Phys., 74, 1095-1098, 2006.

Priest, E. and Forbes, T.: Magnetic Reconnection: MHD Theory and Applications, Cambridge Univ. Press, Cambridge, UK, 2000.

Pritchett, P. L. and Coroniti, F. V.: Localized convection flows and field-aligned current generation in a kinetic model of the nearEarth plasma sheet, Geophys. Res. Lett., 27, 3161-3164, 2000.

Pritchett, P. L. and Coroniti, F. V.: A kinetic ballooning/interchange instability in the magnetotail, J. Geophys. Res., 115, A06301, doi:10.1029/2009JA014752, 2010.

Roberts, K. V. and Taylor, J. B.:Magnetohydrodynamic equations for finite Larmor radius, Phys. Rev. Lett., 8, 197-198, 1962.

Samtaney, R., Loureiro, N. F., Uzdensky, D. A., Schekochihin, A. A., and Cowley, S. C.: Formation of Plasmoid Chains in Magnetic Reconnection, Phys. Rev. Lett., 103, 105004, doi:10.1103/PhysRevLett.103.105004, 2009.

Schnack, D. D.: Lectures in Magnetohydrodynamics: With an Appendix on Extended MHD, Springer, Berlin, 47 pp., 2009.

Shay, M., Drake, J., Rogers, B., and Denton, R.: Alfvénic collisionless magnetic reconnection and the Hall term, J. Geophys. Res., 106, 3759-3772, 2001.

Shay, M. A., Drake, J. F., Swisdak, M., and Rogers, B. N.: The scaling of embedded collisionless reconnection, The scaling of embedded collisionless reconnection, Phys. Plasmas, 11, 21992213, 2004.

Shepherd, L. S. and Cassak, P. A.: Comparison of secondary islands in collisional reconnection to Hall reconnection, Phys. Rev. Lett., 105, 015004, doi:10.1103/PhysRevLett.105.015004, 2010.

Shibata, K. and Tanuma, S.: Plasmoid-induced-reconnection and fractal reconnection, Earth Planet. Space, 53, 473-482, 2001.

Somov, B. V.: Plasma Asrophysics, Part I, Astrophysics and Space Science Library, Springer 2006, 340, 240 pp., 2006.

Sonnerup, B. U. O. and Laird, M. J.: On magnetospheric interchange instability, J. Geophys. Res., 68, 131-139, 1963.

Sweet, P. A.: The neutral point theory of solar flares, in: Electromagnetic Phenomena in Cosmical Physics, edited by: Lehnert, B., 123-134, Cambridge Univ. Press, London, 1958.

Uchida, Y.: New aspects about solar flares revealed by the widedynamic range, high-cadence observations from Yohkoh, Adv. Space Res., 17, 19-28, 1996.

Uchida, Y. and Sakurai, T.: Heating and reconnection of the emerging magnetic flux-tubes and the role of the interchange instability, Solar Phys., 51, 413-429, 1977.

Uzdensky, D. A. and Kulsrud, R. M.: Two-dimensional Numerical Simulation of the Resistive Reconnection Layer, Phys. Plasmas, 7, 4018-4030, 2000.

Winske, D.: Regimes of the magnetized Rayleigh-Taylor instability, Phys. Plasmas, 3, 3966-3974, 1996.

Yamada, M., Kulsrud, R., and Ji, H.: Magnetic reconnection, Rev. Modern Phys., 82, January-March, 2010. 